\title{
Modeling the changing spot features of SW Lacertae: A three year study ${ }^{\star}$
}

\author{
B. Albayrak ${ }^{1}$, G. Djurašević ${ }^{2}$, S. Erkapić 2 , and T. Tanrıverdi ${ }^{1}$ \\ 1 Ankara University, Faculty of Science, Dept. of Astronomy and Space Sciences, 06100 Tandoğan, Ankara, Turkey \\ 2 Astronomical Observatory, Volgina 7, 11160 Belgrade, Serbia and Montenegro and Isaac Newton Institute of Chile, \\ Yugoslav branch, Yugoslavia
}

Received 1 April 2003 / Accepted 18 March 2004

\begin{abstract}
In the present study, the activity of the eclipsing binary of the W UMa-type system SW Lac is examined by analysing the new $B V$ photoelectric observations obtained in 2001, 2002 and 2003 at the Ankara University Observatory. During this period, the seasonal light curves show significant differences and asymmetries. A simultaneous analysis of the light curves is made using Djurašević's inverse-problem method. To explain the light-curve asymmetries and the different maximum light levels in the seasonal light curves we used a Roche model that involved regions containing spots on the components. The analysis shows that SW Lac is in an overcontact configuration with a relatively high degree of overcontact $f_{\text {over }} \sim 31 \%$. The Roche model with spotted areas on the more massive and cooler component yields a good fit of the observations for the whole set of the seasonal light curves, without any changes of the basic system parameters. This indicates that the complex nature of the light-curve variations during the examined period can be explained by the changes of spotted areas on the cooler component, which cover a significant part of the stellar surface.
\end{abstract}

Key words. stars: binaries: eclipsing - stars: binaries: close - stars: individual: SW Lac

\section{Introduction}

The short-period $(P \sim 0.32)$ W UMa-type eclipsing binary SW Lac $\left(V_{\max }=88^{\mathrm{m}} 91, \mathrm{HD} 216598, \mathrm{BD}+37^{\circ} 4717\right.$, HIP 113052) has been extensively observed because of the variability in the light curve and period since its discovery by Miss Ashall (Leavitt 1918). The first photometric observations were made by Brownlee (1957), who reported a variable and asymmetric light curve for the system. The pecularity had been noticed by all other observers (e.g. Bookmyer 1965; Chou 1963; Rucinski 1968; Muthsam \& Rakos 1974; Leung et al. 1984; Niarchos 1987; Lafta \& Grainer 1985; Pena et al. 1993; Jeong et al. 1994; Pribulla et al. 1999). The light curve variation of the system has been interpreted as being produced by the existence of active spot region(s) on the system's components (Stepien 1980; Binnendijk 1984; Leung et al. 1984; Eaton 1986; Jeong et al. 1994; Lee et al. 1991; Djurasević \& Erkapić 1997; Pribulla et al. 1999). The period variation of the system is explained by Panchatsaram \& Abhyankar (1981) and Pribulla et al. (1999) as due to orbital motion caused by a third and fourth component in the system. Hendry \& Mochnacki (1998) detected a late-type third component in the spectrum

Send offprint requests to: $\mathrm{B}$. Albayrak,

e-mail: albayrak@astro1.science.ankara.edu.tr

* Table 1 is only available in electronic form at CDS via anonymous ftp to cdsarc.u-strasbg.fr $(130.79 .128 .5)$ or via http://cdsweb.u-strasbg.fr/cgi-bin/qcat?J/A+A/420/1039 of SW Lac. The contribution of the additional components to the light curve of SW Lac is negligible (Pribulla et al. 1999). Infrared observations were obtained by Jameson \& Akınc1 (1979). Eaton (1983, 1986), Rucinski (1984), and Jeong et al. (1994) analysed IUE observations of the binary.

The spectral types of the component stars of SW Lac were classified as $G 3 V+G 3 V$ by Wood (1980). From a radial velocity study, Struve (1949) determined a mass ratio of $q=m_{\mathrm{c}} / m_{\mathrm{h}}=$ 1.17. The indices $(\mathrm{h}, \mathrm{c})$ refer to the hotter (less massive) secondary and cooler (more massive) primary component, respectively. The most recent radial velocity observations yielded a revised mass ratio of $q=m_{\mathrm{c}} / m_{\mathrm{h}}=1.255$ (Zhai \& Lu 1989).

The aim of the present paper is to analyse the seasonal (2001, 2002 and 2003) $B$ and $V$ light curves with a fixed revised mass ratio of $q=m_{\mathrm{c}} / m_{\mathrm{h}}=1.255$, to study the activity of the system, and to estimate the orbital and physical parameters of SW Lac.

\section{New observations}

The $B V$ observations of SW Lac were carried out on two nights (Oct. 5 and 12) in 2001, one night (Oct. 18) in 2002, and two nights (Oct. 3 and 22) in 2003 at the Ankara University Observatory, using an uncooled SSP-5A photometer attached to a $30-\mathrm{cm}$ Maksutov telescope. The stars BD $+37^{\circ} 4715$ and $\mathrm{BD}+37^{\circ} 4711$ were used as comparison and check stars, respectively. A total of 235, 181 and 405 observations was obtained in each passband for observing seasons of 2001, 2002 

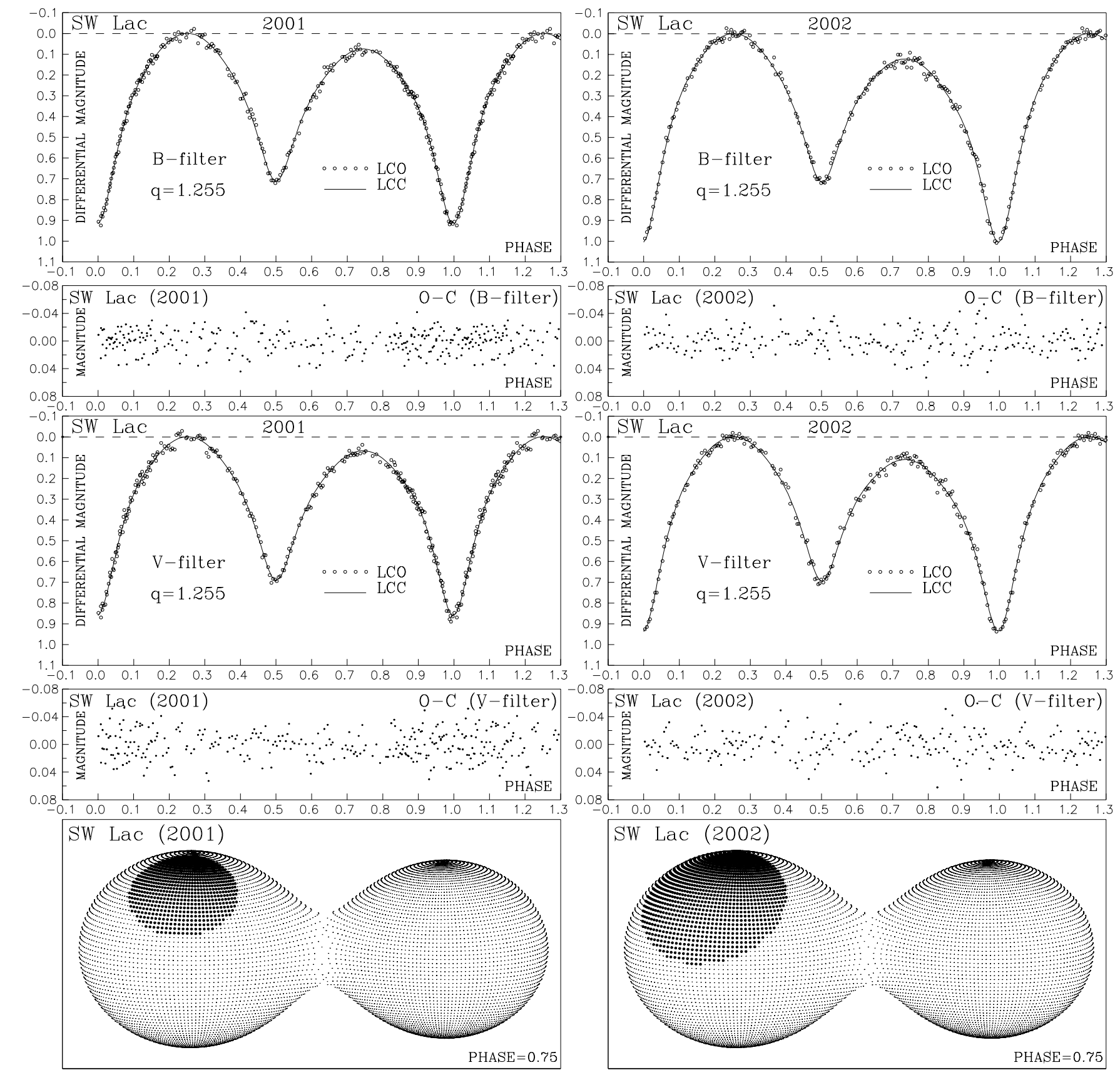

Fig. 1. Observed (LCO) and final synthetic (LCC) light curves of SW Lac with final O-C residuals obtained by analysing seasonal (the left panel for 2001 and the right panel for 2002) $B$ and $V$ observations, and the view of the system at orbital phase 0.75 , obtained with the parameters estimated by analysing the observations. The reference light level of the 2002 light curve at orbital phase 0.25 , which is very probably free of spot effects, is shown by the dashed line.

and 2003, respectively. The nightly extinction coefficients for each passband were determined by using the observations of the comparison star. The probable error of a single observation point was estimated to be \pm 0.026 in both $B$ and $V$ passbands for the 2001 and 2002 seasons, and \pm 0.017 in $B$ and \pm 0.020 in $V$ for the 2003 season. The instrumental differential $B$ and $V$ magnitudes in the sense variable minus comparison corrected for the atmospheric extinction, are given in Table 1 (accessible in electronic form at CDS). The differential $B$ and $V$ light curves obtained in the three observing seasons are shown in Figs. 1 and 2 . There is a significant asymmetry in the light curves, where the light at phase 0.25 as max I is brighter than that at phase 0.75 as max II.

Three ( 2 primary and 1 secondary), two ( 1 primary and 1 secondary), and four ( 2 primary and 2 secondary) minima times for each passband were calculated from the 2001, 2002, and 2003 observations, respectively, using the method of Kwee \& van Woerden (1956). The first mentioned has already been published by Albayrak et al. (2002), while the others are given in Table 2. The most recent study of the period changes of SW Lac was made by Derekas et al. (2002). Their study based on eclipse timings taken from 1990 to 2001 revealed a 


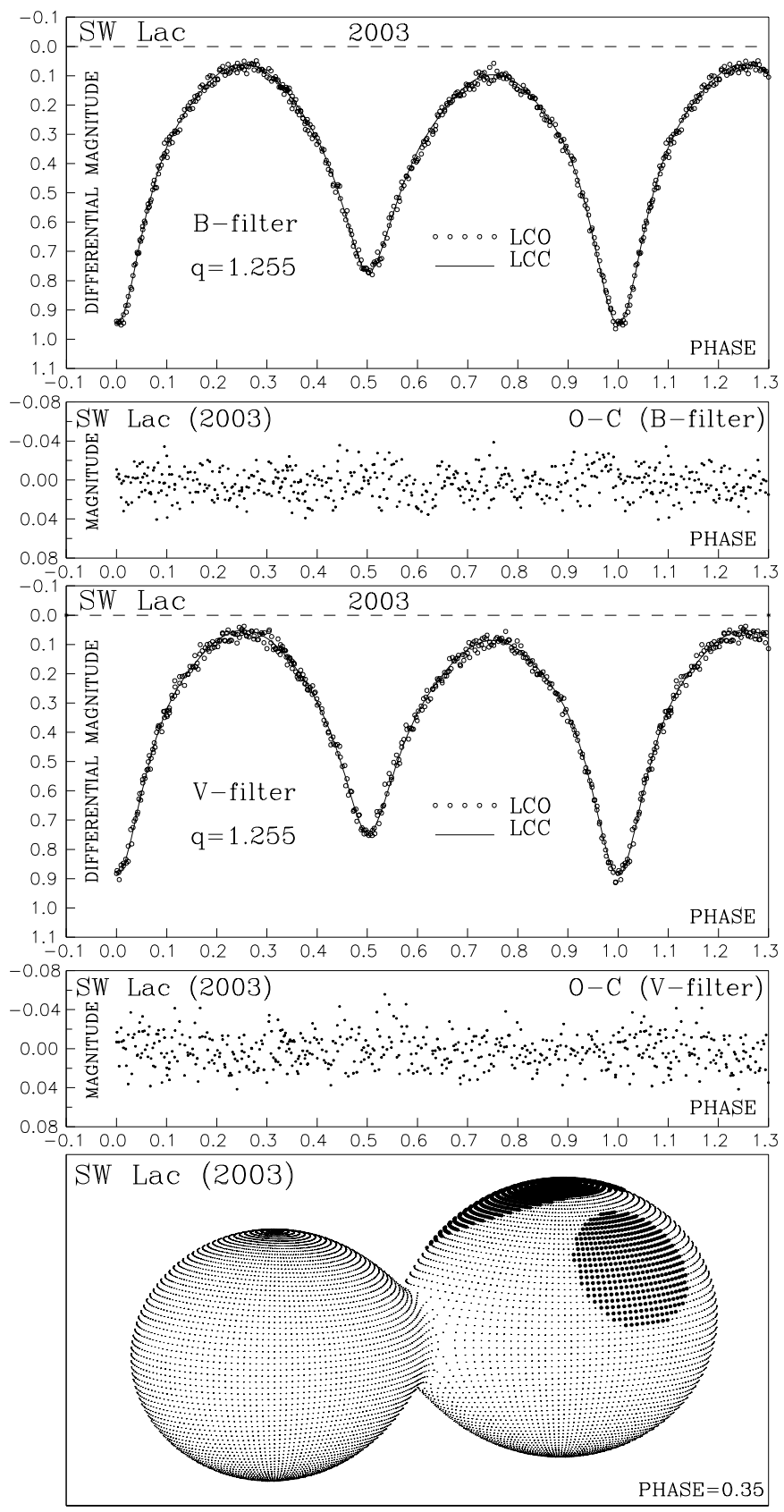

Fig. 2. Observed (LCO) and final synthetic (LCC) light curves of SW Lac with final $\mathrm{O}-\mathrm{C}$ residuals obtained by analysing seasonal (2003) $B$ and $V$ observations and the view of the system at orbital phase 0.35 , obtained with the parameters estimated by analysing the observations. The reference light level of the 2002 light curve at orbital phase 0.25 , which is very probably free of spot effects, is shown by the dashed line.

continuously decreasing period for the system. Thus, by using only our minima times to compute the phase of the observations, we determined the following light elements:

HJD MinI $=2451056.2839+0.3207152 \times E$.

$$
\pm 16 \quad \pm 3
$$

Because of the variable period of SW Lac, the new linear elements will be valid only for a few years around 2003.
Table 2. The minima times obtained in 2002 and 2003 seasons.

\begin{tabular}{ccccc}
\hline \hline & HJD & Error & Min type & Filter \\
\hline $2452566+$ & & & & \\
& 0.3720 & 0.0003 & II & $B$ \\
& 0.3717 & 0.0003 & II & $V$ \\
& 0.5303 & 0.0002 & I & $B$ \\
$2452905+$ & 0.5301 & 0.0003 & I & $V$ \\
& 0.3683 & 0.0002 & II & $B$ \\
& 0.3675 & 0.0001 & II & $V$ \\
$2452916+$ & 0.5279 & 0.0001 & I & $B$ \\
& 0.5278 & 0.0001 & I & $B$ \\
& 0.2729 & 0.0001 & II & $B$ \\
& 0.2725 & 0.0001 & II & $V$ \\
& 0.4324 & 0.0001 & I & $B$ \\
& 0.4320 & 0.0001 & I & $V$ \\
\hline
\end{tabular}

\section{Light curve analysis}

We analyzed the curves using the synthetic light curve programme by Djurašević (1992a) modified for contact configurations (Djurašević et al. 1998). The programme is based on the Roche model and the principles arising from the paper by Wilson \& Devinney (1971). The light-curve analysis was made by applying the inverse-problem method (Djurašević 1992b) based on Marquardt's (1963) algorithm.

The filling parameters of our model include the "spatial filling factor", $F_{\mathrm{h}, \mathrm{c}}=R_{\mathrm{h}, \mathrm{c}} / R_{\text {Roche }_{\mathrm{h}, \mathrm{c}}}$, where $R_{\text {Roche }_{\mathrm{h}, \mathrm{c}}}$ is the polar radius of the critical Roche lobe and $R_{\mathrm{h}, \mathrm{c}}$ is the modeled value, and the "fill-out", $f$, is defined through dimensionless $\Omega$ equipotentials as:

$f_{\text {over }}[\%]=100 \cdot\left(\Omega_{\mathrm{h}, \mathrm{c}}-\Omega_{\mathrm{i}}\right) /\left(\Omega_{\mathrm{o}}-\Omega_{\mathrm{i}}\right)$,

where $\Omega_{\mathrm{h}, \mathrm{c}}, \Omega_{\mathrm{i}}$, and $\Omega_{\mathrm{o}}$ are the potentials of the common photosphere and of the inner and outer contact surfaces, respectively.

In the light curve analysis, the mass ratio of the components was fixed at $q=m_{\mathrm{c}} / m_{\mathrm{h}}=1.255$, estimated by Zhai \& $\mathrm{Lu}$ (1989) from the radial velocity solution. Based on the spectral type (G3V; Wood et al. 1980) the temperature of the less massive (hotter) component, $T_{\mathrm{h}}$, was set at $5630 \mathrm{~K}$.

The present analysis yields $F_{\mathrm{h}}>1$ for the filling coefficient in the critical Roche lobe, i.e., there is an overcontact configuration.

The intensity and angular distribution of the radiation of elementary cells are determined from the stellar effective temperature, limb-darkening, gravity-darkening and from the effect of reflection in the system.

Following Lucy (1967), Rucinski (1969) and Rafert \& Twigg (1980), the gravity-darkening exponents of the stars, $\beta_{\mathrm{h}, \mathrm{c}}$, and their albedos, $A_{\mathrm{h}, \mathrm{c}}$, were set at the values of 0.08 and 0.5 , respectively, appropriate for stars with convective envelopes.

A non-linear limb-darkening law has been used in the present work to avoid the possible negative influence of the wrong evaluation of limb-darkening coefficients on other parameters in the inverse problem. In agreement with 
Claret's (2000) paper, whose tables we used, we chose the new approximation which can be written as:

$$
\begin{aligned}
\frac{I(\mu)}{I(1)}= & 1-a_{1}\left(1-\mu^{1 / 2}\right)-a_{2}(1-\mu)-a_{3}\left(1-\mu^{3 / 2}\right) \\
& -a_{4}\left(1-\mu^{2}\right)
\end{aligned}
$$

where $a_{1,2,3,4}$ are the limb-darkening passband specific coefficients and $\mu=\cos \gamma . I(1)$ is the passband specific intensity at the center of the stellar disc, and $\gamma$ is the angle between the line of sight and the emergent flux. This law, based on the Least-Squares Method, is able to describe the intensity distribution over the whole stellar disk very well, with very good flux conservation. The values of the passband limb-darkening coefficients are derived from the current values of the stellar effective temperature $T_{\text {eff }}$ and surface gravity $\log g$ in each iteration, by interpolation for both of these quantities in Claret's (2000) tables. This was achieved by bi-linear interpolation (Press et al. 1992).

For a successful application of this model in the analysis of the observed light curves, the inverse-problem method proposed by Djurašević (1992b) was used. Optimum model parameters are obtained through the minimization of $\Sigma(\mathrm{O}-\mathrm{C})^{2}$, where $\mathrm{O}-\mathrm{C}$ is the residual between the observed (LCO) and synthetic (LCC) light curves for a given orbital phase. The minimisation of $\Sigma$ is done in an iterative cycle of corrections of the model parameters by using the modified Marquardt (1963) algorithm. In this way, the inverse-problem method provides estimates of the system parameters and their standard errors.

The presence of spots (cool or hot) was used to model the asymmetries in the light curves. In our code these active regions are approximated by circular spots, characterised by the temperature contrast between the spot and the surrounding photosphere $\left(A_{\mathrm{S}}=T_{\mathrm{S}} / T_{*}\right)$, by the angular dimension (radius) of the spot $\left(\theta_{\mathrm{S}}\right)$ and by the longitude $\left(\lambda_{\mathrm{S}}\right)$ and latitude $\left(\varphi_{\mathrm{S}}\right)$ of the spot centre. For more details see e.g. Djurašević et al. (1998).

Since the stars in the system have external convective envelopes, which can exhibit magnetic activity, we started the "spotted solution" by assuming that the components of SW Lac have cool spots, of the same nature as solar magnetic spots. Moreover, since the results of the light curve analysis depend on the choice of the adopted working hypothesis, the analysis was carried out within the framework of several hypotheses with spots on the components. Finally, on the basis of the simultaneous analysis of seasonal $B$ and $V$ light curves, we have chosen the Roche model with dark spots on the more massive (cooler) component as the optimum solution for modeling the asymmetry and depressions in SW Lac light curves. The cooler component has a deeper convective layer which means that more intense active regions can be expected.

The analysis of the asymmetry and deformation of the seasonal light curves of SW Lac presents a problem. Due to the lack of light curves that are free of spot effect we cannot reliably estimate the reference light level representing the immaculate configuration. The highest light level is found in the 2002 light curves at the orbital phase 0.25 . In the further analysis this maximum light level is taken as the reference level with the spot effect contamination most probably at the minimum.
The rest of the light curves are scaled to this light level, which is denoted by a dashed line in Figs. 1 and 2.

The simultaneous analysis of the 2002 light curves gave us the estimates for the basic system parameters, and for the parameters of the active cool spot region which deforms the light curves. In the analysis of these seasonal light curves, the inclination of the orbit was estimated to be $i \sim 79.8$, the filling coefficient for the critical Roche lobe $F_{\mathrm{h}} \sim 1.06$ and the temperature of the cooler (more massive) star $T_{\mathrm{c}} \sim 5348 \mathrm{~K}$.

The system's basic parameters, obtained by analysing the 2002 seasonal light curves, were fixed in the inverse-problem solution for other, more or less deformed and asymmetrical light curves. In the subsequent analysis, these light curves are normalised to the reference light level of the 2002 light curves at orbital phase 0.25 , and their analysis was made with optimising the spot parameters. Since one cannot exclude in advance the possibility of a change of basic system parameters, they are also included in the iterative procedure of optimisation in the final iterations. As we expected, these parameters remained unchanged, with the exception of relatively small changes in the temperature of the secondary.

The analysis shows that the 2001 and 2002 seasonal light curves can be successfully fit by using the Roche model with one spot on the more massive (cooler) component. In addition to asymmetry, the 2003 light curves also exhibit a conspicious depression of the light level compared to the reference maximum light level in the year 2002 (dashed line in Figs. 1 and 2). To interpret these light curves we had to provide the Roche model with two active cool spot regions. With this model we obtained a good fit of the 2003 seasonal light curve without any changes of the basic system parameters. The unchanged values of the basic system parameters and the good quality of the fit for all three seasonal light curves are proof that the hypothesis in which the spots were placed on the more massive component was well chosen.

Also, this indicates that the nature of the light-curve variations during the examined period can be basically explained by the changes in the spot areas across the stellar surfaces.

\section{Results and discussion}

The parameters derived from the light-curve analysis are listed in Table 3. The first three rows of the table present the number of observations $n$, the final sum of squares of residuals between observed (LCO) and synthetic (LCC) light curves

$\sum_{i=1}^{n}\left(\mathrm{O}_{i}-\mathrm{C}_{i}\right)^{2}$,

and the standard deviation of the observations

$\sigma=\sqrt{\frac{\sum_{i=1}^{n}\left(\mathrm{O}_{i}-\mathrm{C}_{i}\right)^{2}}{(n-1)}}$.

In the same table the spot characteristics (spot temperature factor, $A_{\mathrm{S}}=T_{\mathrm{S}} / T_{\mathrm{c}}$; angular radius, $\theta_{\mathrm{S}}$; longitude, $\lambda_{\mathrm{S}}$; latitude, $\varphi_{\mathrm{S}}$; spot area in percents of the stellar surface on which the spots are located, $\left.\sigma_{\mathrm{S} 1, \mathrm{~S} 2}[\%]\right)$ are also given. The determination of these parameters is based on the simultaneous fitting 
Table 3. Results of the analysis of the SW Lac seasonal light curves obtained by solving the inverse problem for the Roche model including active regions on the more massive (cooler) component.

\begin{tabular}{|c|c|c|c|}
\hline Quantity & 2001 & 2002 & 2003 \\
\hline$n$ & 470 & 362 & 810 \\
\hline$\Sigma(\mathrm{O}-\mathrm{C})^{2}$ & 0.1624 & 0.1342 & 0.1976 \\
\hline$\sigma$ & 0.0186 & 0.0193 & 0.0156 \\
\hline$q=m_{\mathrm{c}} / m_{\mathrm{h}}$ & 1.255 & 1.255 & 1.255 \\
\hline$f_{\mathrm{h}, \mathrm{c}}$ & 1.0 & 1.0 & 1.0 \\
\hline$\beta_{\mathrm{h}, \mathrm{c}}$ & 0.08 & 0.08 & 0.08 \\
\hline$A_{\mathrm{h}, \mathrm{c}}$ & 0.5 & 0.5 & 0.5 \\
\hline$T_{\mathrm{h}}$ & 5630 & 5630 & 5630 \\
\hline$A_{\mathrm{S} 1}=T_{\mathrm{S} 1} / T_{\mathrm{c}}$ & $0.84 \pm 0.01$ & $0.85 \pm 0.01$ & $0.87 \pm 0.01$ \\
\hline$\theta_{\mathrm{S} 1}$ & $31.8 \pm 0.5$ & $44.4 \pm 0.4$ & $46.9 \pm 0.4$ \\
\hline$\lambda_{\mathrm{S} 1}$ & $278.9 \pm 1.6$ & $294.3 \pm 1.1$ & $263.6 \pm 1.3$ \\
\hline$\varphi_{\mathrm{S} 1}$ & $48.3 \pm 1.6$ & $45.4 \pm 1.1$ & $61.9 \pm 1.2$ \\
\hline$\sigma_{\mathrm{S} 1}[\%]$ & $\sim 7$ & $\sim 14$ & $\sim 16$ \\
\hline$A_{\mathrm{S} 2}=T_{\mathrm{S} 2} / T_{\mathrm{c}}$ & & & $0.89 \pm 0.01$ \\
\hline$\theta_{\mathrm{S} 2}$ & & & $26.4 \pm 0.4$ \\
\hline$\lambda_{\mathrm{S} 2}$ & & & $87.8 \pm 1.6$ \\
\hline$\varphi_{\mathrm{S} 2}$ & & & $30.5 \pm 2.7$ \\
\hline$\sigma_{\mathrm{S} 2}[\%]$ & & & $\sim 5$ \\
\hline$T_{\mathrm{c}}$ & $5347 \pm 12$ & $5348 \pm 11$ & $5345 \pm 13$ \\
\hline$F_{\mathrm{h}}$ & $1.059 \pm 0.001$ & $1.060 \pm 0.001$ & $1.060 \pm 0.001$ \\
\hline$i\left[^{\circ}\right]$ & $79.85 \pm 0.11$ & $79.85 \pm 0.14$ & $79.85 \pm 0.09$ \\
\hline$a_{1}^{\mathrm{h}, \mathrm{c}}(B)$ & $+0.5308,+0.5979$ & $+0.5308,+0.5977$ & $+0.5308,+0.5983$ \\
\hline$a_{2}^{\mathrm{h}, \mathrm{c}}(B)$ & $-0.3755,-0.6945$ & $-0.3754,-0.6934$ & $-0.3754,-0.6964$ \\
\hline$a_{3}^{\mathrm{h}, \mathrm{c}}(B)$ & $+1.3274,+1.7057$ & $+1.3274,+1.7045$ & $+1.3273,+1.7077$ \\
\hline$a_{4}^{\mathrm{h}, \mathrm{c}}(B)$ & $-0.5963,-0.7035$ & $-0.5963,-0.7032$ & $-0.5963,-0.7039$ \\
\hline$a_{1}^{\mathrm{h}, \mathrm{c}}(V)$ & $+0.5750,+0.6261$ & $+0.5750,+0.6260$ & $+0.5750,+0.6264$ \\
\hline$a_{2}^{\mathrm{h}, \mathrm{c}}(V)$ & $-0.2306,-0.5050$ & $-0.2305,-0.5041$ & $-0.2305,-0.5067$ \\
\hline$a_{3}^{\mathrm{h}, \mathrm{c}}(V)$ & $+0.9634,+1.3431$ & $+0.9634,+1.3418$ & $+0.9634,+1.3453$ \\
\hline$a_{4}^{\mathrm{h}, \mathrm{c}}(V)$ & $-0.4920,-0.6255$ & $-0.4919,-0.6251$ & $-0.4919,-0.6262$ \\
\hline$\Omega_{\mathrm{h}, \mathrm{c}}$ & 3.9801 & 3.9796 & 3.9795 \\
\hline$f_{\text {over }}[\%]$ & 30.86 & 30.95 & 30.96 \\
\hline$R_{\mathrm{h}}[D=1]$ & 0.357 & 0.357 & 0.357 \\
\hline$R_{\mathrm{c}}[D=1]$ & 0.395 & 0.395 & 0.395 \\
\hline$L_{\mathrm{h}} /\left(L_{\mathrm{h}}+L_{\mathrm{c}}\right)(B ; V)$ & $0.538 ; 0.530$ & $0.548 ; 0.540$ & $0.557 ; 0.548$ \\
\hline $\mathcal{M}_{\mathrm{h}}\left[M_{\odot}\right]$ & $0.78 \pm 0.03$ & & \\
\hline $\mathcal{M}_{\mathrm{c}}\left[M_{\odot}\right]$ & $0.98 \pm 0.02$ & & \\
\hline $\mathcal{R}_{\mathrm{h}}\left[R_{\odot}\right]$ & $0.92 \pm 0.02$ & & \\
\hline $\mathcal{R}_{\mathrm{c}}\left[R_{\odot}\right]$ & $1.02 \pm 0.02$ & & \\
\hline $\log g_{\mathrm{h}}$ & $4.40 \pm 0.02$ & & \\
\hline $\log g_{\mathrm{c}}$ & $4.41 \pm 0.02$ & & \\
\hline$M_{\mathrm{bol}}^{\mathrm{h}}$ & $5.07 \pm 0.03$ & & \\
\hline$M_{\mathrm{bol}}^{\mathrm{c}}$ & $5.09 \pm 0.05$ & & \\
\hline$a_{\text {orb }}\left[R_{\odot}\right]$ & $2.375 \pm 0.009$ & & \\
\hline
\end{tabular}

Note: $n$ - number of observations, $\Sigma(\mathrm{O}-\mathrm{C})^{2}-$ final sum of squares of residuals between observed (LCO) and synthetic (LCC) light curves, $\sigma$-standard deviation of the observations, $q=m_{\mathrm{c}} / m_{\mathrm{h}}$ - mass ratio of the components, $f_{\mathrm{h}, \mathrm{c}}, \beta_{\mathrm{h}, \mathrm{c}}, A_{\mathrm{h}, \mathrm{c}}-$ nonsynchronous rotation coefficients, gravity-darkening exponents and albedo of the components, $T_{\mathrm{h}, \mathrm{c}}$ - temperature of the hotter primary and cooler secondary, $A_{\mathrm{S} 1,2}, \theta_{\mathrm{S} 1,2}, \lambda_{\mathrm{S} 1,2}$ and $\varphi_{\mathrm{S} 1,2}$ - spots' temperature coefficient, angular dimension, longitude and latitude (in arc degrees), $\sigma_{\mathrm{S} 1, \mathrm{~S} 2}[\%]-$ spot area in percents of the stellar surface on which the spots are located, $F_{\mathrm{h}, \mathrm{c}}-$ filling factors for the critical Roche lobe of the hotter (less massive) and cooler (more massive) star, $i\left[^{\circ}\right]$ - orbit inclination (in degrees), $a_{1}^{\mathrm{h}, \mathrm{c}}, a_{2}^{\mathrm{h}, \mathrm{c}}, a_{3}^{\mathrm{h}, \mathrm{c}}, a_{4}^{\mathrm{h}, \mathrm{c}}-$ nonlinear $(B, V)$ limb-darkening coefficients of the components (Claret's formula), $\Omega_{\mathrm{h}, \mathrm{c}}-$ dimensionless surface potentials of the primary and secondary, $f_{\text {over }}[\%]=100 \cdot\left(\Omega_{\mathrm{h}, \mathrm{c}}-\Omega_{\mathrm{in}}\right) /\left(\Omega_{\mathrm{out}}-\Omega_{\mathrm{in}}\right)-$ degree of overcontact, $R_{\mathrm{h}, \mathrm{c}}$ - polar radii of the components in units of the distance between the component centres, $L_{\mathrm{h}} /\left(L_{\mathrm{h}}+L_{\mathrm{c}}\right)-(B ; V)$ luminosity of the hotter star (including spot on the cooler one), $\mathcal{M}_{\mathrm{h}, \mathrm{c}}\left[M_{\odot}\right], \mathcal{R}_{\mathrm{h}, \mathrm{c}}\left[R_{\odot}\right]-$ stellar masses and mean radii of stars in solar units, log $g_{\mathrm{h}, \mathrm{c}}-\log \operatorname{lorithm}$ (base 10) of the mean surface acceleration (effective gravity) for system stars, $M_{\text {bol }}^{\mathrm{h}, \mathrm{c}}-$ absolute bolometric magnitudes of SW Lac components, and $a_{\text {orb }}\left[\mathcal{R}_{\odot}\right]$ - orbital semi-major axis in units of solar radius. 
of the available light curves in the $B$ and $V$ photometric bands for three epochs of the observations with the same set of basic system parameters. The relative area of the circular spot is calculated assuming that the shape of the star is spherical, and it is only a rough approximation of the real situation. This value gives us the ratio of the spot surface to the stellar surface, and can be used as an indicator of the spot activity. Finally, in Table 3 we present some important absolute parameters. They are derived from the mass ratio of the components $q=m_{\mathrm{c}} / m_{\mathrm{h}}=1.255$, estimated by Zhai \& Lu (1989) from a radial velocity study.

The errors of the parameter estimates arise from the nonlinear least-squares method on which the inverse-problem method is based. The uncertainties of these parameters may be larger than we estimated. Having in mind the errors of the input parameters of the model, which are treated as fixed in the inverse problem, we estimate that the errors of the parameters will definitely be larger (approximately 2 times).

The solution for each seasonal individual light curve is presented in Figs. 1 and 2, where the optimum fit of the observed light curves (LCO) to the synthetic ones (LCC) is shown. The $\mathrm{O}-\mathrm{C}$ residuals between the observed (LCO) and optimum synthetic (LCC) light curves are given too. The figure also includes the view of Roche models for observing seasons (2001, 2002 and 2003) separately. Using such plots, one can see how a close binary system would appear at a certain orbital phase, chosen so that the spots are visible.

It is evident from Table 3 and from Figs. 1 and 2 that the Roche model with the spot areas on the cooler component gives a satisfactory fit of the seasonal light curves.

Moreover, several attempts were made to fit the observations by assuming a spot model with spots on both components. In that case we can obtain fits which are similar in quality, but for this we need some changes of system basic parameters derived by analysing the individual seasonal light curves. We consider that case as less reliable, so that we feel that it should probably be excluded.

The size of the spot area can be used as an indicator of the system activity. The analysis of the asymmetric light curves from 2001 shows the presence of a smaller spotted area $\left(\sigma_{\mathrm{S} 1} \sim\right.$ $7 \%$ ), located relatively close to the stellar polar region. The analysis of the light curves from 2002 gives a larger spot on the same stellar hemisphere, which covers a significant part of the stellar surface $\left(\sigma_{\mathrm{S} 1} \sim 14 \%\right)$. So, their presence leads to a conspicuous asymmetry in the light curves. It appears that the system's activity was higher in 2002. In the year 2003, the light curves are less asymmetrical, but they exhibit a large depression of the maximum light level compared to the reference level in 2002. Modeling the 2003 light curves without changing basic system parameters requires a more complex Roche model with two spot regions on the secondary, which is an indication of the increased activity of the system during the year 2003. The location of the large spot $\left(\sigma_{\mathrm{S} 1} \sim 16 \%\right)$, near the stellar polar region, has not changed significantly compared to the previous two seasons but the spot is somewhat larger. The additional small spot $\left(\sigma_{\mathrm{S} 2} \sim 5 \%\right)$ is located at almost opposite longitude and at moderate latitude. These two spots cover approximately $21 \%$ of the surface of the secondary, and show that the system activity is larger than in the two previous seasons.

\section{Conclusions}

Both presentations of our results obtained in the analysis of SW Lac light curves in 2001, 2002 and 2003 (Table 3 and Figs. 1 and 2) show that the Roche model with the spot areas on the cooler component provides a satisfying fit of the observations. From the whole period the main variations in the light curves can be explained by the development and migration of spot regions on the cooler component. The spot area shows a continous increase during the whole examined period. The solutions obtained by solving the inverse problem do not show changes of the system basic parameters among the different seasonal light curves. Consequently, the complex nature of the light-curve variations may be attributed to variable starspot activity.

The model can successfully reproduce the observed light curves which cover an interval of 3 years. Our analysis shows that during the deeper (primary) minimum the cooler (more massive and larger) component eclipses the hotter (less massive and smaller) one. The solutions presented here indicated that SW Lac is in an overcontact configuration with a relatively high degree of overcontact $f_{\text {over }} \sim 31 \%$, with a small temperature difference between the components $\left(\Delta T=T_{\mathrm{h}}-T_{\mathrm{c}} \sim 280 \mathrm{~K}\right)$ indicating thermal contact between the components.

Having in mind the large changes in the shape of the light curves, future continuous photometric observations of this interesting system are of great interest. To monitor the development of active regions at stellar surface and to establish the possible cyclic nature of the system activity, photometric observations of SW Lac should be carried out for at least 10 years. These observations together with the analysis of the orbital period variations could then offer a relatively reliable estimate of the evolution of SW Lac.

Acknowledgements. This work has been supported by the Turkish Academy of Sciences, in the framework of the Young Scientist Award Program (BA/TÜBA-GEBİP/2001-2-2), and by the Ministry for Sciences and Technology of Serbia through the project 1191 "Stellar physics". This research has made use of the Simbad database, operated at CDS, Strasbourg, France. We appreciate the anonymous referee for her/his constructive and generous comments.

\section{References}

Albayrak, B., Tanrıverdi, T., \& Aydın, C. 2002, IBVS, No. 5300

Binnendijk, L. 1984, PASP, 96, 646

Bookmyer, B. B. 1965, AJ, 70, 415

Brownlee, R. R. 1957, ApJ, 1235, 327

Chou, K. C. 1963, AJ, 68, 342

Claret, A. 2000, A\&A, 363, 1081

Derekas, A., Kiss, L. L., \& Bebesi, Z. S. 2002, IBVS, No. 5255

Djurašević, G. 1992a, Ap\&SS, 196, 241

Djurašević, G. 1992b, Ap\&SS, 197, 17

Djurašević, G., \& Erkapić, S. 1997, Bull. Astron. Belgrade, No. 155, 55

Djurašević, G., Zakirov, M., Hojaev, A., \& Arzumanyants, G. 1998, A\&AS, 131, 17 
Eaton, J. A. 1983, ApJ, 268, 800

Eaton, J. A. 1986, Acta Astron., 36, 79

Hendry, H. D., \& Mocnacki, S. W. 1998, AJ, 504, 978

Jameson, R. F., \& Akınci1, R. 1979, MNRAS, 188, 421

Jeong, J. H., Kang, Y. W., Lee, W. B., \& Sung, E. C. 1994, ApJ, 421, 779

Kwee, K. K., \& van Woerden, H. 1956, Bull. Astr. Inst. Neth., 12, 327

Lafta, S., \& Grainer, J. F. 1985, Ap\&SS, 114, 23

Lee, W. B., Sung, E. C., \& Kang, Y. W. 1991, PASA, 9, 297

Leavitt, H. 1918, Harvard Obs. Cir., No. 207

Leung, K.-C., Zhai, D., \& Zhang, R. 1984, PASP, 96, 634

Lucy, L. B. 1967, Zs. f. Ap., 65, 89

Marquardt, D. W. 1963, J. Soc. Ind. Appl. Math., 11(2), 431

Muthsam, H., \& Rakos, K. D. 1974, A\&AS, 13, 127

Niarchos, P. G. 1987, A\&AS, 67, 365

Panchatsaram, T., \& Abhyankar, K. D. 1981, Bull. Astron. Soc. India, 9,31

Pena, J. H., Hobart, M. A., \& Rodriguez, E. 1993, Rev. Mex. Astron. Astrofis., 25, 63
Press, W. H., Teukolsky, S. A., Vetterling, W. T., \& Flannery, B. P. 1992, Numerical Recipes in Fortran, The Art of Scientific Computing, Second Edition (New York: Cambridge University Press), 120

Pribulla, T., Chochol, D., \& Parimucha, S. 1999, Contr. Astron. Obs. Skalnate Pleso, 29, 111

Rafert, J. B., \& Twigg, L. W. 1980, MNRAS, 139, 78

Rucinski, S. M. 1968, Acta Astron., 18, 49

Rucinski, S. M. 1969, Acta Astron., 19, 245

Rucinski, S. M., Brunt, C. C., \& Pringle, J. E. 1984, MNRAS, 208, 309

Stepien, K. 1980, Acta Astron., 30, 315

Struve, O. 1949, ApJ, 109, 436

Wilson, R. E., \& Devinney, E. J. 1971, ApJ, 166, 605

Wood, F. B., Oliver, J. P., Florkowski, D. R., \& Koch, R. H. 1980, A Finding List for Observers of Interacting Binary Stars, Publications of the University of Pensilvania, Astronomical Series, XII

Zhai, D. S., \& Lu, W. X. 1989, Chin. Astron. Astrophys., 13, 350 